\author{
M. MERANO \\ S. SONDEREGGER \\ A. CROTTINI \\ S. COLLIN \\ E. PELUCCHI \\ P. RENUCCI \\ A. MALKO \\ M.H. BAIER \\ E. KAPON \\ J.D. GANIÈRE \\ B. DEVEAUD
}

\section{Time-resolved cathodoluminescence of InGaAs/AlGaAs tetrahedral pyramidal quantum structures}

Institute of Quantum Electronics and Photonics, Ecole Polytechnique Fédérale de Lausanne, 1015 Lausanne, Switzerland

\section{Received: 20 December 2005 \\ Published online: 14 June 2006 • () Springer-Verlag 2006}

ABSTRACT An original time resolved cathodoluminescence set up has been used to investigate the optical properties and the carrier transport in quantum structures located in InGaAs/AlGaAs tetrahedral pyramids. An InGaAs quantum dot formed just below the top of the pyramid is connected to four types of low-dimensional barriers: InGaAs quantum wires on the edges of the pyramid, InGaAs quantum wells on the (111)A facets and segregated AlGaAs vertical quantum wire and AlGaAs vertical quantum wells formed at the centre and at the pyramid edges. Experiments were performed at a temperature of $92 \mathrm{~K}$, an accelerating voltage of $10 \mathrm{kV}$ and a beam probe current of $10 \mathrm{pA}$. The cathodoluminescence spectrum shows five luminescence peaks. Rise and decay times for the different emission wavelengths provide a clear confirmation of the peak attribution (previously done with other techniques) to the different nanostructures grown in a pyramid. Moreover, experimental results suggest a scenario where carriers diffuse from the lateral quantum structures towards the central structures (the InGaAs quantum dot and the segregated $\mathrm{AlGaAs}$ vertical quantum wire) via the InGaAs quantum wires on the edges of the pyramid. According to this hypothesis, we have modeled the carrier diffusion along these quantum wires. An ambipolar carrier mobility of $1400 \mathrm{~cm}^{2} / \mathrm{V} \mathrm{s}$ allows to obtain a good fit to all temporal dependences.

PACS 78.67.-n

1

\section{Introduction}

A complete characterization of a nanostructure involves the investigation of its structural, electronic and optical properties. Different experimental tools for testing the structure of a quantum dot (QD) or a quantum wire (QWR) are employed. We might cite direct imaging methods, such as scanning tunnelling microscopy (STM), atomic force microscopy (AFM), transmission electron microscopy (TEM) and scanning electron microscopy (SEM) [1]. STM and AFM have in principle atomic resolution and, as well as SEM, are able to reveal directly the morphology of a surface. TEM is used to inspect a nanostructure embedded in its nano-environment.

Fax: +33(0)1 693199 96, E-mail: michele.merano@ensta.fr
Electronic and optical properties of a single nanostructure are mainly studied with spectroscopic tools. Existing local luminescence probe techniques are [2]: spatially localized micro-photoluminescence spectroscopy ( $\mu$-PL) using either strong focusing or masking; near-field optical microscopy (SNOM) to avoid the diffraction limitation of far-field optics; cathodoluminescence (CL), using focused energetic electrons in an electron microscope; and scanning tunneling luminescence (STL), using low-energy electrons injected or extracted from the tip of a STM.

When combined with picosecond or femtosecond laser pulses, spectroscopic techniques allow for studying the carriers dynamics in a semiconductor sample. Phenomena as different as carrier capture, energy relaxation, radiative recombination or carrier transport are then accessible [3].

In this article we employ an original time resolved cathodoluminescence (TRCL) set-up to investigate the time resolved luminescence of quantum structures located in InGaAs/AlGaAs tetrahedral pyramids [4]. Because of its high spatial resolution $(50 \mathrm{~nm})$ and its high temporal resolution (12 ps), this set-up is successful in characterizing the different nanostructures grown in a single pyramid and it allows for describing the carrier transport in a system where QWs, QWRs and a QD coexist.

\section{Experimental set up}

Picosecond cathodoluminescence is an original TRCL set up (for complete description and characterization see [5, 6]). A home-made high brightness picosecond electron gun [7] replaces the thermionic electron gun of a commercial JEOL 6360 SEM (Fig. 1). Electron pulses of 12 ps temporal width and $80.7 \mathrm{MHz}$ repetition rate are focused on a probe diameter of $50 \mathrm{~nm}$ onto the sample. The deflection coil in front of the last microscope lens scans the electron probe in a raster across the specimen. The secondary electrons (SE), produced as a consequence of the primary beam-specimen interaction, are collected by an Everhart-Thornley detector. The latter is connected to a computer that, in synchronism with the scanning electron beam, reproduces a topographic image of the sample.

The luminescence signal is collected by a parabolic mirror and focused either on the entrance slit of a monochromator 


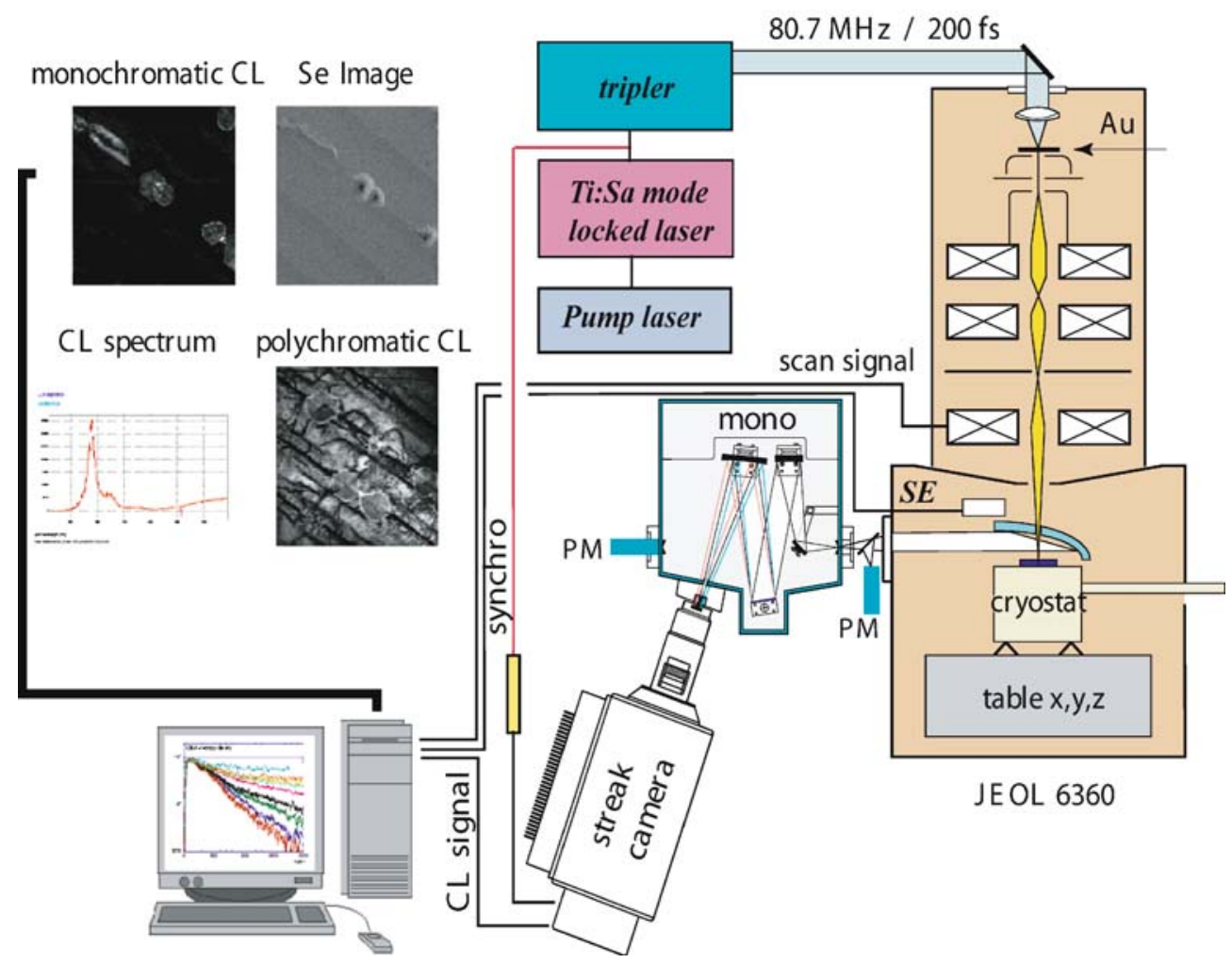

FIGURE 1 Picosecond cathodoluminescence set up. A photoemissionbased electron gun replaces the thermionic electron gun of the SEM. Ultraviolet light pulses are focused on a gold photocathode in order to produce electron pulses. Examples of SE and CL imaging modes are reported. A streak camera is used to record a spectrum and to perform temporal analysis

or directly on a photomultiplier (PM). The photomultiplier signal is first amplified and then sent to the computer that displays a polychromatic CL image of the sample. A monochromatic image is obtained when the PM is placed after the monochromator exit slit.

Once we have obtained a SE and a CL image of the system under investigation (a nanostructure for instance) we can proceed to study the luminescence emitted from different points of it. It is sufficient to work in the spot mode of the electron microscope. The electron beam is not scanned anymore on the sample surface but is rather directed on the point of interest. A streak camera is used to record spectra and to perform temporal analysis.

Finally, a $\mathrm{LN}_{2}$ cryostat allows the sample to be cooled down to $90 \mathrm{~K}$. It consists of a cold copper finger in contact with the sample holder. Despite its simplicity, this system guarantees a great stability.

\section{$3 \quad$ Sample growth and structure}

The investigated pyramidal nanostructures are fabricated with the following procedure. Prior to growth, a GaAs substrate is patterned with a $5 \mu \mathrm{m}$ pitch hexagonal matrix of tetrahedral recesses, using standard photolithography and wet chemical etching. The characteristic tetrahedral pyramid pattern, exposing (111)A facets, is obtained in this case due to the anisotropic nature of the wet chemical etching.

Low pressure organometallic vapor deposition growth of InGaAs/AlGaAs heterostructures is then performed on the patterned substrate. The higher AlGaAs OMCVD growth rate on the (111)A facets leads to the formation of a very sharp corner and wedges $(<10 \mathrm{~nm}$ radius of curvature) with self limiting profiles at the centre and the wedges of the pyramid. The first epitaxial layer $\left(\mathrm{Al}_{0.75} \mathrm{Ga}_{0.25} \mathrm{As}\right.$ nominal thickness $45 \mathrm{~nm}$ ) is followed by $130 \mathrm{~nm}$ of $\mathrm{Al}_{0.55} \mathrm{Ga}_{0.45}$ As. Subsequently $140 \mathrm{~nm} \mathrm{Al} \mathrm{Al}_{0.30} \mathrm{Ga}_{0.70}$ As with a $0.5 \mathrm{~nm} \operatorname{In}_{0.10} \mathrm{Ga}_{0.90} \mathrm{As}$ layer inserted at its centre and other $130 \mathrm{~nm}$ of $\mathrm{Al}_{0.55} \mathrm{Ga}_{0.45} \mathrm{As}$ are grown. Finally, $10 \mathrm{~nm}$ of GaAs capping layer are added. All thicknesses refer to growth on planar (100) substrates. The actual growth rate at the tip of the pyramid can be significantly higher (by a factor of 3 to 5), and is actually governed by a complex interplay among surface diffusion on the (111)B planar surfaces, growth rate anisotropies on the different crystal planes, capillarity diffusion and entropy of mixing effects $[4,8-10]$.

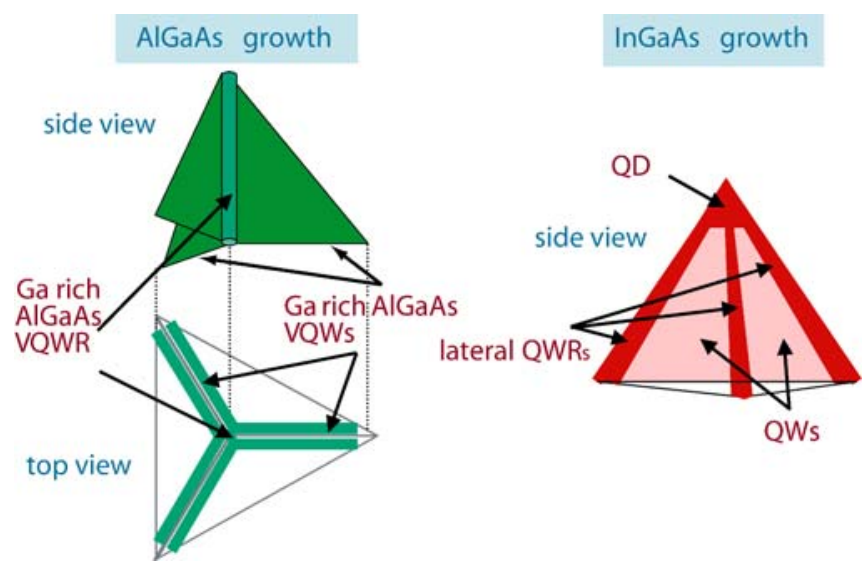

FIGURE 2 Schematic representation of the five heterostructures present in an InGaAs/AlGaAs pyramid. The InGaAs nanostructures are sandwiched between two $\mathrm{Al}_{0.30} \mathrm{Ga}_{0.70} \mathrm{As}$ barrier layers. Ga rich VQWR and VQWs selforder respectively at the centre and at the pyramid edges 
The growth process results in the formation of an InGaAs quantum dot (QD) connected to several types of lowdimensional barriers: InGaAs quantum wires (QWRs) on the edges of the pyramid, InGaAs quantum wells (QWs) on the (111)A facets and segregated $\sim \mathrm{Al}_{0.04} \mathrm{Ga}_{0.96}$ As vertical quantum wire (VQWR) and $\sim \mathrm{Al}_{0.20} \mathrm{Ga}_{0.80}$ As vertical quantum wells (VQWs) formed at the centre and at the pyramid edges (Fig. 2).

\section{Experimental results \\ 4.1 Luminescence spectrum}

TRCL experiments on InGaAs/AlGaAs pyramidal quantum structures were performed at a temperature of $90 \mathrm{~K}$. The accelerating voltage was $10 \mathrm{kV}$ and the exciting current was $10 \mathrm{pA}$ i.e., $\sim 1$ electron per pulse. Vacuum in the microscope specimen chamber was $10^{-5}$ mbar. Spectra were recorded with a slit width of $200 \mu \mathrm{m}$ and a 600 grooves $/ \mathrm{mm}$ monochromator grating. For our monochromator this correspond to a resolution of $2 \mathrm{~nm}(4 \mathrm{meV})$ at a wavelength of $800 \mathrm{~nm}$. This value is largely sufficient for our purposes; at a temperature of $90 \mathrm{~K}$ we expect indeed a thermal broadening of $7.5 \mathrm{meV}$. The CL spectrum was recorded with a thermoelectric cooled CCD that we used at the place of the streak camera (Fig. 1).

The spectral features of the nanostructures under investigation extend from 1.93 to $1.48 \mathrm{eV}$ (Fig. 3). The spectrum reported on Fig. 3 was integrated for $500 \mathrm{~ms}$. It has been obtained while the electron beam was scanning over a field covered by one pyramid. The scan speed was the standard one that is equivalent to $142 \mathrm{~ms}$ per image. We clearly see five peaks. The one at the minimum energy $(1.52 \mathrm{eV})$ will be associated with the QD. Its FWHM is $6.7 \mathrm{meV}$. The energy spread observed for it is consistent with the sample temperature. Holes states in the dot are indeed separated by a few $\mathrm{meV}$ and at temperatures between 80 to $100 \mathrm{~K}$ its emission lines broaden. The peaks at $1.57,1.66,1.77,1.90 \mathrm{eV}$ correspond respectively to the VQWR, QWR, VQW, and the QW. We will discuss in the

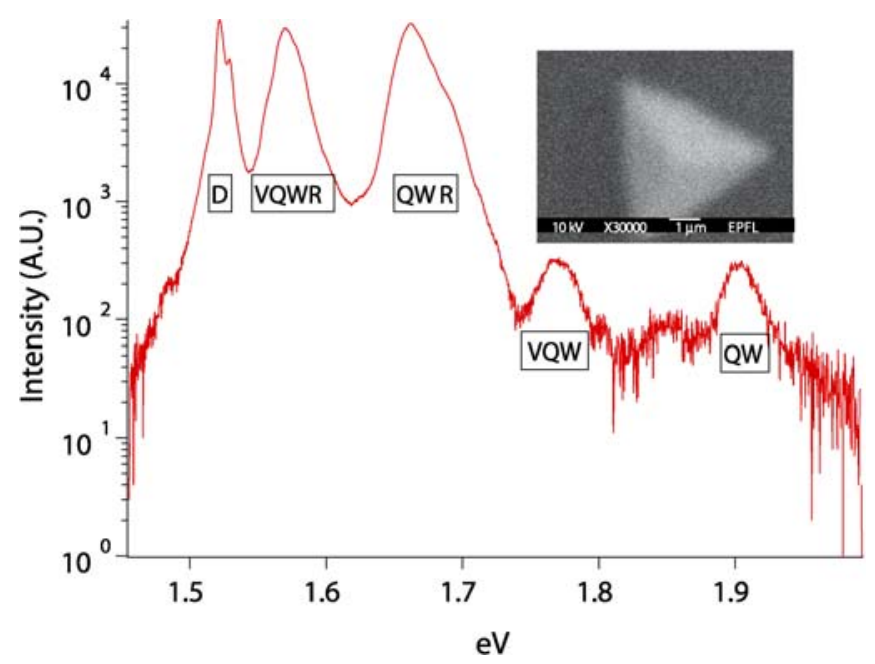

FIGURE 3 Luminescence spectrum from InGaAs/AlGaAs pyramidal quantum structures. Five peaks are clearly visible. They correspond to the five nanostructures formed in the pyramid. Inset: Image of the surface scanned by the electron beam while the spectrum was taken next sections how the spectral features of each nanostructure can be identified and we will see that TRCL can be very useful for this task.

\subsection{Spectrally resolved CL images}

Spectrally resolved CL images of the pyramids are shown in Fig. 4. We registered an image for each of the five spectral peaks. Contrast and brightness were optimized for each picture to better evidence the emission pattern at the different wavelengths. All the images reported are from the same region of area $25 \mu \mathrm{m} \times 30 \mu \mathrm{m}$. CL imaging mode is useful to directly identify the $1.90 \mathrm{eV}$ emission line with the InGaAs QWs grown on the (111) A facets. Emission from one facet looks uniform while pyramid edges and centre are dark.

Wavelength dispersive CL images do not allow for other attributions. The spectral features corresponding to the different nanostructures grown in such pyramids were originally assigned with other techniques. Features at 1.57 and $1.77 \mathrm{eV}$ can be attributed to the VQWR and the VQW because they also appear at the same energy in the control sample in which no InGaAs QW layer was grown [4]. Aluminium segregation in the VQWR is expected to be stronger than in the VQW so the line at $1.57 \mathrm{eV}$ is attributed to the VQWR. The CL image centred at $1.77 \mathrm{eV}$ confirms that the VQW emits at this energy. The InGaAs heterostructures can be identified by their energy position and the expected InGaAs thickness, as determined using a simple square well model, neglecting lateral quantization [11]. Several other experiments, like excitation power dependent measurements, showing a strong saturation at high powers for the QD contribution, cleaved edge meas-

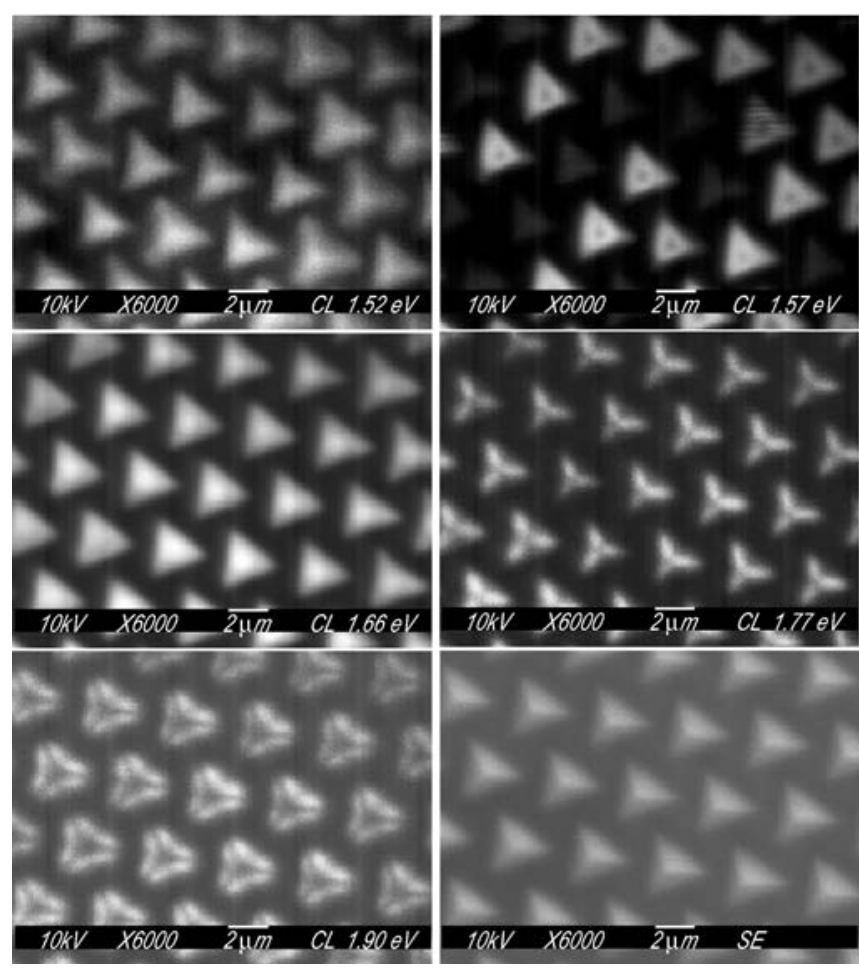

FIGURE 4 SE and monochromatic CL images from the same region of the sample. From top to bottom CL images centred at the QD, VQWR, QWR, VQW, QW emission wavelengths and the SE image used as reference 
urements and microPL line scans, were used to further corroborate these peak assignments [4]. It was also observed that the QD emission evolves as expected with dot thickness, with the ground state transition energy increasing with decreasing the dot thickness [12].

Our CL images relative to QD, QWR and VQWR can not be related in a direct way with the nanostructure location at the origin of the luminescence signal. This is typical for a CL experiment because the emission pattern does not depend only on the primary electron diffusion range but also on the carrier transport processes involved in these complex nanostructures. The carriers diffusion length so limits the spatial resolution of the wavelength dispersive image. If we consider for instance the CL image at the QD emission line $(1.52 \mathrm{eV})$, we observe that the emission pattern corresponds to the entire pyramid. This means that carriers find a path to diffuse towards the dot from all points of the pyramid. Another reason for the complex CL image patterns is the fact that recombination sites trap carriers that could potentially emit light at the considered wavelength. These potential traps appear as dark regions in the corresponding spectrally-resolved CL image.

Carrier mobility increases as a function of the temperature of the sample. Low temperature $(10 \mathrm{~K})$ spectrally resolved CL images of a slightly different sample, GaAs/AlGaAs heterostructures grown in the same regular matrix of tetrahedral recesses, are reported in literature. They were obtained with a standard continuous cathodoluminescence system [13].

5

\section{Time resolved measurements}

Time resolved experiments were carried out according to the following procedure. We excited with the electron pulses six different points on one pyramid and for each emission wavelength we studied the temporal response. The chosen points were: the top of the pyramid, the middle and the bottom of the pyramid edge, and three points on the pyramid facet. A colour code will be used to distinguish the luminescence emission from the different points.

When this first experiment was carried out, the JEOL 6360 electron microscope was not yet provided with the spot mode function so we centred on the computer display the point to be analysed and we increased the microscope magnification to its maximum possible value $(300000 \times)$. In this way the excitation region was a square of $300 \mathrm{~nm} \times 300 \mathrm{~nm}$ i.e., of the order of the excitation volume.

\subsection{QW emission}

The temporal dependence of the QWs (parallel to the three tetrahedron faces) luminescence is reported on Fig. 5. The continuous CL image, shown on Fig. 4 and reported also in the inset of Fig. 5, suggests that the luminescence decay times for the QWs depend on the excitation point. The top and the edges of the pyramid look dark because of

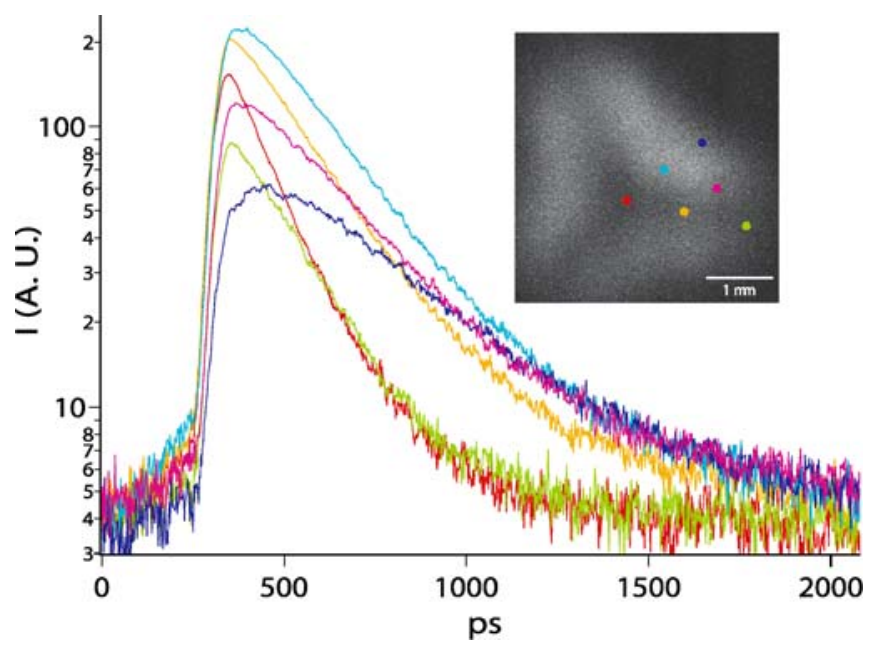

FIGURE 5 Temporal response of the luminescence signal emitted by the QW (emission acquired at $1.90 \mathrm{eV}$ ). Colours correspond to the different excitation points. Inset: CL image at the corresponding emission wavelength. The excitation points for the different temporal profiles are reported

carrier capture in the other heterostructures. For excitation in these regions carriers from the QWs are trapped in the QWRs, VQWs, VQWR or the QD. As a consequence of this, we expect the QW decay time to be shorter at these points. Indeed if we compare the different excitation points (see Table 1) we find that the decay time is $130 \mathrm{ps}$ when we excite the top of the pyramid and it is 300-400 ps when the bottom of the facet is excited.

Rise times are also interesting. They are defined as the time for the signal to rise from $10 \%$ to $90 \%$ of the maximum value. In this case the rise time represents the carrier capture from the AlGaAs barrier into the InGaAs QWs. Since the QWs are all grown on the (111)A facet, we expect them to be equal. Our experimental results validate this scenario.

\subsection{VQW emission}

TRCL experiments (Fig. 6) confirm that the luminescence signal at $1.77 \mathrm{eV}$ is emitted by the VQWs created by the growth process above and below the three corner QWRs. AlGaAs VQWs are formed (see Fig. 2) at the pyramid edges. We expect that carriers diffuse more rapidly towards the VQWs when we excite a point on the pyramid corner than when we direct the electron beam on a facet.

If we look at the rise and decay times at this wavelength, we observe that the rise times do not vary much. Instead, the decay times of the luminescence profiles increase with the distance of the exciting point from the pyramid edge, while the maxima of the intensity emission decrease. We interpret the rise time as the time the carriers take to leave the VQW because of carrier trapping in the QWR. The decay times represent here the carrier capture in the VQW and are mainly due

\begin{tabular}{lrrrrrr}
\hline & red & orange & green & cyan & magenta & blue \\
\hline $\begin{array}{l}\text { rise time } 10 \%-90 \%(\mathrm{ps}) \\
\text { decay time }(\mathrm{ps})\end{array}$ & 56 & 55 & 58 & 57 & 57 & 100 \\
\hline
\end{tabular}

TABLE 1 QW rise and decay times for different excitation points 


\begin{tabular}{lrrrrrr}
\hline & red & orange & green & cyan & magenta & blue \\
\hline rise time $10 \%-90 \%(\mathrm{ps})$ & 51 & 55 & 53 & 68 & 65 & $\sim 100$ \\
decay time $(\mathrm{ps})$ & 190 & 260 & 130 & 330 & 340 & \\
\hline
\end{tabular}

TABLE 2 VQW rise and decay times for different excitation points It is not possible to evaluate the decay time relative to the blue point

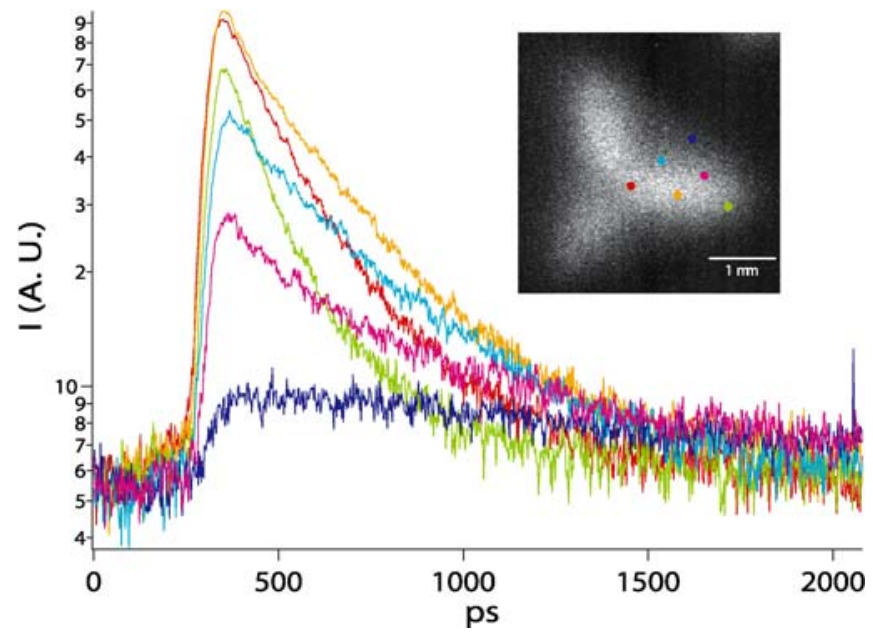

FIGURE 6 Temporal response of the luminescence signal emitted by the VQW (emission centred at $1.77 \mathrm{eV}$ ). Inset: CL image at the corresponding emission wavelength. The excitation points for the different temporal profiles are reported

to carrier diffusion. Indeed, when carriers need more time to access the quantum structure than they need to leave it, the temporal response shows the arrival time as a decay time and the leaving time as a rise time. This also assumes very short trapping times in the different quantum structures, in very good agreement with other measurements [14].

With a $10 \mathrm{kV}$ primary excitation beam, electron-holes pairs are created within a volume of $300 \mathrm{~nm}$ [15] around the point of impact of the electrons i.e., mostly in the AlGaAs barriers of the structure. If we also consider the fact that in our case the primary electron beam scans a surface of $300 \mathrm{~nm} \times$ $300 \mathrm{~nm}$ we can safely assume an electron-hole creation volume with a radius of $400 \mathrm{~nm}$. Taking the decay times observed for the excitation points on the edge of the pyramid this imply a diffusion coefficient of the order of $10 \mathrm{~cm}^{2} / \mathrm{s}$ corresponding to a mobility of of the order of $1000 \mathrm{~cm}^{2} / \mathrm{V} \mathrm{s}$ at $90 \mathrm{~K}$. This value seems indeed quite reasonable (see below).

\subsection{QWR emission}

InGaAs QWRs are located along the pyramid wedges. We observe a difference in the rise time of the luminescence signal when the electron pulses are focused on the pyramid corner or on the pyramid facet (Fig. 7).

Rise times for the QWR are now clearly not relaxation limited. If we consider the three points along the pyramid edge, we explain this result by the carrier capture from the

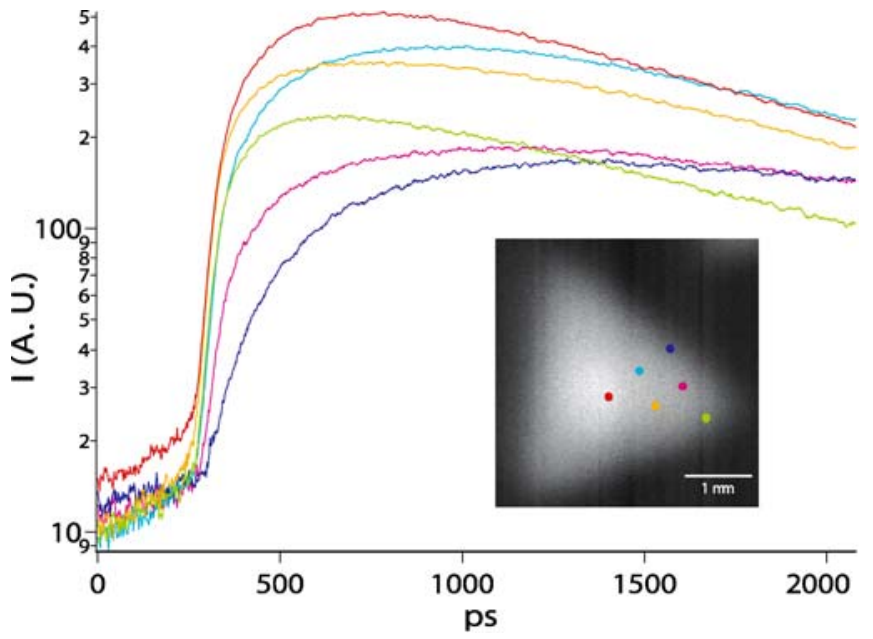

FIGURE 7 Temporal response of the luminescence signal emitted by the QWR (emission centred at $1.66 \mathrm{eV}$ ). Inset: CL image at the corresponding emission wavelength. The excitation points for the different temporal profiles are reported

$400 \mathrm{~nm}$ excitation volume. For the three excitation points on the pyramid facet we have to consider the path that carriers travel to get trapped into the QWR. The luminescence decay time is also longer with respect to the wells. We could estimate it only for the edge excitation points because in the other cases the signal is too long. The decay time that we observe is due to two important contributions: the carrier lifetime in the QWR and the carrier diffusion towards the central structures (see below).

\subsection{VQWR emission}

Data for the VQWR created by the growth process along the axis of the pyramid are shown in Fig. 8. The measured rise times confirm that the VQWR is a central structure. Indeed rise times increase with the excitation point distance from the pyramid centre because carriers have to travel a longer path to get there. Notice that this is the first case where we observe a clear variation of the rise times luminescence correlated with the position of the excitation points along the pyramid edges (Table 4).

It is important to observe that for all excitation points, except the one on top of the pyramid, the luminescence signal still increases after $2 \mathrm{~ns}$. When we consider the lateral structures, the only luminescence signal that is not zero in the time interval between 1.5 and $2 \mathrm{~ns}$ is the QWR emission. The temporal response of the wells decays more rapidly as compared

\begin{tabular}{lrrrrrr}
\hline & red & orange & green & cyan & magenta & blue \\
\hline $\begin{array}{l}\text { rise time } 10 \%-90 \%(\mathrm{ps}) \\
\text { decay time }(\mathrm{ps})\end{array}$ & 250 & 230 & 180 & 350 & 470 & 640 \\
\hline
\end{tabular}

TABLE 3 QWR rise and decay times for different excitation points. It is not possible to evaluate the decay time relative to the cyan, magenta and blue points 


\begin{tabular}{lcccccc}
\hline & red & orange & green & cyan & magenta & blue \\
\hline rise time $_{10 \%-90 \%(\mathrm{ps})}$ & 210 & 870 & 1100 & 820 & 1000 & 1300 \\
\hline
\end{tabular}

TABLE 4 VQWR rise times for different excitation points

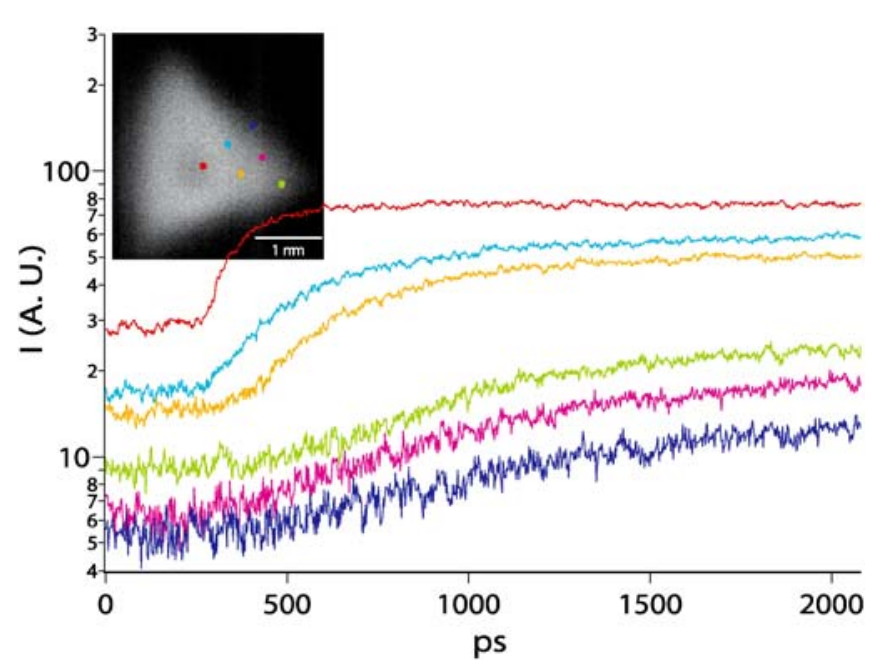

FIGURE 8 Temporal response of the luminescence signal emitted by the VQWR (emission centred at $1.57 \mathrm{eV}$ ). Inset: $\mathrm{CL}$ image at the corresponding emission wavelength. The excitation points for the different temporal profiles are reported

with the response of the other structures. This observation imposes clearly the scenario that carriers diffuse towards the central structures via the QWRs. Later in this article we will develop a model that uses and confirms this hypothesis.

\section{5}

\section{QD emission}

The QD emission is saturated. Its luminescence is constant in time. As we saw in the previous section the VQWR continues to emit for a very long time. Carriers are still there even after $12 \mathrm{~ns}$. As long as carriers are present in the VQWR, they can diffuse towards the QD and saturate it.

A way to avoid this problem is to reduce the temperature of the sample. Results published in literature show that the decay times for these quantum wires decrease considerably with temperature [16]. Other measurements will be performed when a He cryostat will be mounted in our set-up.

\section{6}

\section{Carrier transport}

In the previous section, we noticed that for all excitation points, except the one on top of the pyramid, the luminescence emission from the VQWR is still increasing even $2 \mathrm{~ns}$ after the electron pulse excitation. At the same time if we look at the lateral structures, the only luminescence signal that is not zero in the time interval between 1.5 and $2 \mathrm{~ns}$ is the QWR emission. As we said before, this allows us to suppose that carriers diffuse towards the central structures via the QWRs.

We develop here a simple model to describe the carrier transport in InGaAs/AlGaAs pyramidal quantum structures. We show how carrier diffusion in the QWRs can be used to simulate our data. In particular, the model is successful in evaluating the carriers mobility in the QWR and in describing at the same time the population dynamics of the QWR and the VQWR.

\subsection{Diffusion model}

To gain insight into the transport properties of the QWR we focus our attention on the two excitation points located on the pyramid edge plus the excitation point on top of the pyramid. The wire length derived from our images is $1.8 \mu \mathrm{m}$. This value must be corrected by considering the angle $\left(\sim 54^{\circ}\right)$ that the pyramid edge forms with the substrate, giving a QWR length of $3 \mu \mathrm{m}$.

The primary electron beam incident on a pyramid edge creates electron-hole pairs in all its interaction volume, mainly in the AlGaAs barriers. The diffusion coefficients for electrons and holes are generally different. Electrons have a greater mobility with respect to the holes. The charge separation induced by the difference in the diffusion coefficient accelerates holes and decelerates electrons. For this reason we will consider the ambipolar diffusion coefficient to model our data. To simulate all this, according to our discussion earlier, we make the assumption that in our model carriers are created in a reservoir and they need a time $\tau$ to get trapped into the QWR. Carriers in the QWR diffuse in a one dimensional system towards the central structures.

Equation (1) governs the number of carriers per unity length $u(x, t)$ located at time $t$ and at point $x$ in the reservoir

$\frac{\partial u(x, t)}{\partial t}=-\frac{u}{\tau}$.

As discussed earlier $\tau$ is much smaller that the carrier lifetimes of the QWR and the VQWR. For this reason in (1) we neglect the carrier diffusion coefficient in the reservoir.

The initial spatial distribution $u(x, 0)$ centred on the excitation point $C$ is given by

$u(x, 0)=N_{0} \frac{1}{\sqrt{2 \pi} \sigma} \exp \left(-\frac{(x-C)^{2}}{2 \sigma^{2}}\right)$,

where $\sigma=400 \mathrm{~nm}$ and $N_{0}$ fixed the total number of carriers that will be injected into the wire.

The number of carriers per unit length $n(x, t)$ in the QWR obeys the equation

$\frac{\partial n(x, t)}{\partial t}=D \frac{\partial^{2} n(x, t)}{\partial^{2} x}-\frac{n}{\tau_{1}}+\frac{u}{\tau}$,

$n(x, 0)=0$,

where $\tau_{1}$ is the carrier lifetime in the QWR. The diffusion coefficient $D$ is related to the carriers mobility $\mu$ by the Einstein relation

$D=\mu \frac{k T}{e}$,

where $k$ is the Boltzmann constant, $T$ is the sample temperature and $e$ the electron charge. 
As boundary conditions we chose a reflecting barrier at the end of the QWR (point $B$ )

$$
\left.\frac{\partial n(x, t)}{\partial x}\right|_{x=B}=0
$$

and an absorbing barrier at the top of the QWR (point $A$ )

$n(A, t)=0$.

The flux of particles absorbed by $A$ is [17]

$J=-\left.D \frac{\partial n(x, t)}{\partial x}\right|_{x=A}$.

A fraction of these is captured by the VQWR and the rest is reflected back to the QWR.

If we look at the temporal profile of the luminescence emission for the VQWR when we excite the top of the pyramid, we observe that it is rather constant in time (Fig. 8). This fact suggests the hypothesis that it is saturated. (As we will see in a next section our simulations confirm this hypothesis.) In this case we are forced to consider the number of free states in the VQWR when we compute the number $J_{1}$ of carriers trapped by this wire in the time interval $t, t+\mathrm{d} t$

$J_{1} \mathrm{~d} t=-\left.D \frac{\partial n(x, t)}{\partial x}\right|_{x=A}\left(1-\frac{N(t)}{N_{\text {sat }}}\right) \mathrm{d} t$.

In this last equation $N(t)$ is the population in the VQWR, $N_{\text {sat }}$ is the maximum number of carriers that can be captured in the VQWR and $J_{1}$ is proportional to the fraction of VQWR free states.

The rate equation for the VQWR population is

$\frac{\mathrm{d} N(t)}{\mathrm{d} t}=J_{1}-\frac{N(t)}{\tau_{2}}$,

$N(0)=N_{0}$,

where $\tau_{2}$ is the carrier lifetime in the VQWR and $N_{0}$ is the number of electron-hole pairs eventually present in the wire when the next excitation pulse arrives onto the pyramid (see Sect. 5.4).

In order to compare the results of our simulations with the experimental data, we have to compute the number of photons emitted by the QWR $\left(I_{\mathrm{QWR}}\right)$ and the $\mathrm{VQWR}\left(I_{\mathrm{VQWR}}\right)$ in the time interval $t, t+\mathrm{d} t$

$$
\begin{aligned}
& I_{\mathrm{QWR}}(t)=\frac{\int_{\mathrm{QWR}} n(x, t) \mathrm{d} x}{\tau_{1}^{\mathrm{r}}}, \\
& I_{\mathrm{VQWR}}(t)=\frac{N(t)}{\tau_{2}^{\mathrm{r}}},
\end{aligned}
$$

where $\tau_{1}^{\mathrm{r}}$ and $\tau_{2}^{\mathrm{r}}$ are the radiative lifetimes.

\subsection{Simulation results}

The best fit to our results is obtained by setting $\tau=150 \mathrm{ps}, \tau_{1}=\tau_{1}^{\mathrm{r}}=2 \mathrm{~ns}, \tau_{2}=5 \mathrm{~ns}, \tau_{2}^{\mathrm{r}}=7.5 \mathrm{~ns}, \tau_{2}^{\mathrm{nr}}=15 \mathrm{~ns}$, and $\mu=1400 \mathrm{~cm}^{2} / \mathrm{V} \mathrm{s}[18,19]$. Such values would be almost impossible to measure with any other technique. They are
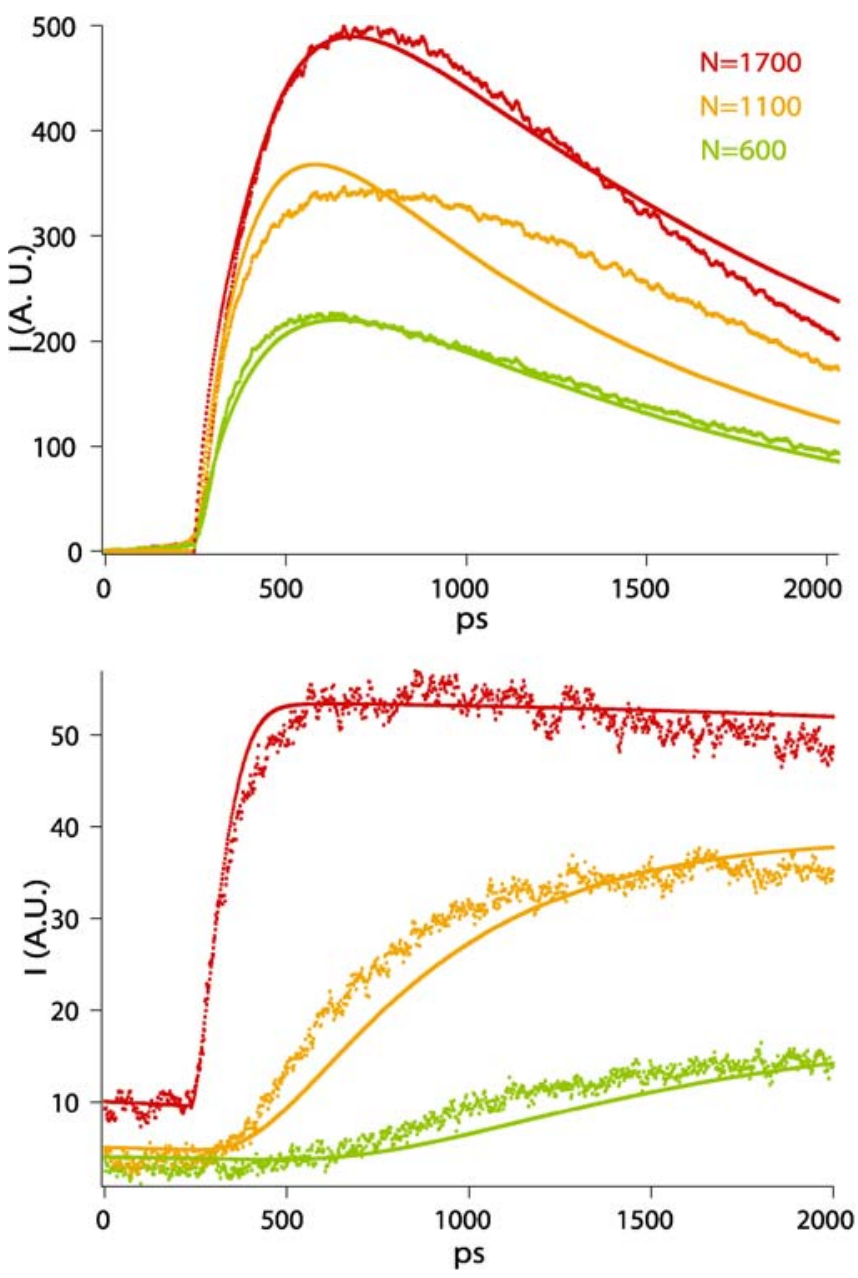

FIGURE 9 Fit of the experimental data in the hypothesis of one population dynamics. Top: QWR, bottom: VQWR

in reasonably well agreement with diffusivities and mobilities observed for example in GaAs/AlGaAs superlattices by $\mathrm{CW}$ and time resolved luminescence. Figure 9 shows the fit for the temporal profile of the luminescence emitted by the QWR. The intensities of the experimental curves differ when we move from an excitation point to another one. We explain this observation with a different number of carriers injected in the QWR in the three cases that we are considering. To take account of this fact we vary the parameter $N_{0}$ in the model to have 1700 carriers injected in the wire when we excite the top of the pyramid and 1100 and 600 for points at the middle and at the bottom of the pyramid edge. In our model the absolute value of $N_{0}$ is totally arbitrary, only ratios between different values of $N_{0}$ are important.

The algorithm we developed also computes the temporal dependence of the VQWR emission (Fig. 9). The fit is very good. In particular it is important to note that we are successful in reproducing the intensity ratios for the VQWR curves once we have set those for the QWR.

\section{$7 \quad$ Conclusion}

TRCL has been used to investigate the optical properties and the carrier transport in quantum structures lo- 
cated in InGaAs/AlGaAs tetrahedral pyramids. An InGaAs quantum dot formed just below the top of the pyramid is connected to several types of low-dimensional barriers: InGaAs quantum wires on the edges of the pyramid, InGaAs quantum wells on the (111)A facets and segregated AlGaAs vertical quantum wire and $\mathrm{AlGaAs}$ vertical quantum wells formed at the centre and at the pyramid edges.

TRCL experiments were performed at a temperature of $92 \mathrm{~K}$, an accelerating voltage of $10 \mathrm{kV}$, and a beam probe current of $10 \mathrm{pA}$. The continuous CL spectrum shows five luminescence peaks. TRCL allows for an unambiguous luminescence peak attribution to the different nanostructures grown in a pyramid. Spectrally resolved CL images clearly identify the peak corresponding to the InGaAs QWs. The CL images relative to QD, VQWR, QWR, and VQW cannot be directly related with the nanostructure location at the origin of the luminescence signals. This is typical for CL experiments because the emission pattern does not depend only on the primary electron diffusion range, but also on the carrier transport and recombination processes in these complex nanostructures.

Time resolved measurements provide valuable information for a safe peak attribution. We have used electron pulses to excite six different points on the pyramid: three located on the pyramid edge and three located on the pyramid facet. Rise and decay times for the different emission energies are strongly dependent on the excitation point location and can be correlated to the carrier capture and relaxation processes in the different nanostructures and to the carrier diffusion mechanism in the pyramid.

In particular, except for the excitation point on top of the pyramid, we observe that the VQWR luminescence signal still increases after $2 \mathrm{~ns}$ (the maximum streak camera temporal window). When we look at the lateral structures, the only luminescence signal that is not zero in the time interval between 1.5 and $2 \mathrm{~ns}$ is that of the QWR. The temporal response of the wells (QW and VQW) falls rapidly to zero with respect to the response of the other structures. This observation, together with the VQWR rise time behaviour, suggests a scenario where carriers move towards the central structures via the QWR.

According to this hypothesis, we have modelled the carrier diffusion along the QWR. We are successful in fitting the luminescence response of the QWR and of the VQWR. The best fit is obtained by assuming the lifetime of the VQWR and QWR to be 5 and 2 ns respectively, and the carrier mobility in the QWR to be $1400 \mathrm{~cm}^{2} / \mathrm{V}$ s. This value is of the same order of the carrier mobility in the AlGaAs barriers, as estimated from the decay time of the VQWs. Our result is similar to experimental diffusion coefficients in QWRs published in literature [20].

ACKNOWLEDGEMENTS We would like to thank Nguyen Hoan and Jean Paul Hervé from OPEA (Vincennes, France) and Roger Rochat and Nicolas Leiser for their help and technical support. This work was strongly supported by the Swiss National Science Foundation, NCCR project "Quantum Photonics".

\section{REFERENCES}

1 D. Bimberg, Quantum Dot Heterostructures (1st ed., Wiley, 1999)

2 A. Gustafsson, M.E. Pistol, L. Montelius, L. Samuelson, J. Appl. Phys. 84, 1715 (1998)

3 J. Shah, Ultrafast Spectroscopy of Semiconductors and Semiconductor Nanostructures (Springer, 1996)

4 A. Hartmann, Y. Ducommun, K. Leifer, E. Kapon, J. Phys.: Condens. Matter 11, 5901 (1999)

5 M. Merano, S. Sonderegger, A. Crottini, S. Collin, P. Renucci, E. Pelucchi, A. Malko, M.H. Baier, E. Kapon, B. Deveaud, J.D. Ganiere, Nature 438, 479 (2005)

6 M. Merano, S. Sonderegger, A. Crottini, S. Collin, P. Renucci, F. Fuzesi, D. Martin, B. Deveaud, J.D. Ganiere, unpublished

7 M. Merano, S. Collin, P. Renucci, M. Gatri, S. Sonderegger, A. Crottini, J.D. Ganiere, B. Deveaud, Rev. Sci. Instrum. 76, 085108 (2005)

8 E. Pelucchi, M. Baier, Y. Ducommun, S. Watanabe, E. Kapon, Phys. Stat. Solidi B 238, 233 (2003)

9 A. Hartmann, L. Loubies, F. Reinhardt, E. Kapon, Appl. Phys. Lett. 71, 1314 (1997)

10 G. Biasiol, E. Kapon, Phys. Rev. Lett. 81, 2962 (1998)

11 Y. Ducommun, Semiconductor Quantum Dots Grown in Inverted Pyramids, PhD thesis, EPFL (2001)

12 E. Kapon, E. Pelucchi, S. Watanabe, A. Malko, M. Baier, K. Leifer, B. Dwir, F. Michelini, M. Dupertuis, Physica E 25, 288 (2004)

13 K. Leifer, A. Hartmann, Y. Ducommun, E. Kapon, Appl. Phys. Lett. 77, 3923 (2000)

14 B. Deveaud, F. Clerot, A. Regreny, K. Fujiwara, K. Mitsunaga, J. Ohta, Appl. Phys. Lett. 55, 2646 (1989)

15 L. Reimer, Scanning Electron Microscope (1st ed., Springer, 1985)

16 D.Y. Oberli, M.A. Dupertuis, F. Reinhardt, E. Kapon, Phys. Rev. B 59 2910 (1999)

17 F. Reif, Statistical and Thermal Physics (McGraw-Hill, 1985)

18 B. Deveaud, A. Chomette, B. Lambert, A. Regreny, R. Romestain, P. Edel, Solid State Commun. 57, 885 (1986)

19 A. Chomette, B. Deveaud, J.Y. Emery, A. Regreny, B. Lambert, Solid State Commun. 54, 75 (1985)

20 V. Emiliani, F. Intonti, C. Lienau, T. Elsaesser, R. Notzel, K.H. Ploog, Phys. Rev. B 64, 155316 (2001) 